\title{
DIAMETER DIMINISHING TO ZERO IFSs
}

\author{
by Radu MICULESCU and Alexandru MIHAIL
}

\begin{abstract}
In this paper we introduce the notion of diameter diminishing to zero iterated function system, study its properties and provide alternative characterizations of it.
\end{abstract}

2010 Mathematics Subject Classification: 28A80, 37C70, 54H20

Key words and phrases: iterated function system (IFS), (hyperbolic) $\varphi$ contractive IFS, (hyperbolic) locally uniformly point fibred IFS, (hyperbolic) uniformly point fibred IFS, IFS having (hyperbolic) attractor, (hyperbolic) diameter diminishing to zero IFS

\section{INTRODUCTION}

The notion of iterated function system, which is due to J. Hutchinson (see [9]), was popularized by M. Barnsley (see [3]). It represents one of the main ways to generate fractal sets and (since it has numerous applications) various generalizations of this concept were introduced. Among them we mention the one exhibited by A. Kameyama (see [11]) under the label of self-similar system which is a topological generalization of the attractor of an iterated function system.

In connection with Kamayema's work, R. Atkins, M. Barnsley, A. Vince and D. Wilson (see [1]) presented a theorem that characterizes hyperbolic affine iterated function systems defined on $\mathbb{R}^{m}$. Results connected with the aforementioned theorem are included in [2], [4], [13], [14], [15], [16], [18], [22] and [23].

Along these lines of research, in this paper, we introduce the concept of (hyperbolic) diameter diminishing to zero iterated function system (see Definition 2.17 and Remarks 2.19) and study its properties (see Propositions $3.1-3.7)$.

In addition, via the concepts of hyperbolic $\varphi$-contractive iterated function system (see Definition 2.11 and Remarks 2.19), hyperbolic (locally) uniformly point fibred iterated function system (see Definitions 2.13 and 2.14 and Remarks 2.19) and iterated function system having hyperbolic attractor (see Definition 2.15 and Remarks 2.19), we provide alternative characterizations of hyperbolic diameter diminishing to zero iterated function systems (see 
Theorem 4.1). Consequently we also come up with different ways to prove that an iterated function system has hyperbolic attractor.

\section{PRELIMINARIES} $B$.

Given two sets $A$ and $B$, by $B^{A}$ we mean the set of functions from $A$ to

By $\mathbb{N}$ we mean the set $\{0,1,2, \ldots, n, \ldots\}$ and by $\mathbb{N}^{*}$ we mean the set $\{1,2, \ldots, n, \ldots\}$.

For a set $X$, a function $f: X \rightarrow X$ and $n \in \mathbb{N}^{*}$, by $f^{[n]}$ we mean the composition of $f$ by itself $n$ times. By $f^{[0]}$ we mean the identity function $I d_{X}: X \rightarrow X$ given by $I d_{X}(x)=x$ for every $x \in X$.

Given a metric space $(X, d)$, by:

- $P_{b}(X)$ we mean the set of non-empty bounded subsets of $X$

- $P_{b, c l}(X)$ we mean the set of non-empty bounded and closed subsets of $X$

- $P_{c p}(X)$ we mean the set of non-empty compact subsets of $X$

- by $B(x, r)$ we mean the set $\{y \in X \mid d(x, y)<r\}$, where $x \in X$ and $r>0$.

\section{The Hausdorff-Pompeiu metric}

Definition 2.1. Given a metric space $(X, d), H_{d}: P_{b, c l}(X) \times P_{b, c l}(X) \rightarrow$ $[0,+\infty)$ given by

$$
\begin{aligned}
& H_{d}(A, B)=\max \left\{\sup _{x \in A} d(x, B), \sup _{x \in B} d(x, A)\right\}= \\
= & \inf \left\{\varepsilon \in[0, \infty) \mid A \subseteq E_{\varepsilon}(B) \text { and } B \subseteq E_{\varepsilon}(A)\right\},
\end{aligned}
$$

for all $A, B \in P_{b, c l}(X)$, where

$$
d(x, A)=\inf _{y \in A} d(x, y)
$$

and

$E_{\varepsilon}(A) \stackrel{\text { def }}{=}\{y \in X \mid$ there exists $x \in A$ such that $d(x, y)<\varepsilon\}=\cup_{x \in A} B(x, \varepsilon)$,

turns out to be a metric, which is called the Hausdorff-Pompeiu metric.

Proposition 2.2. For a metric space $(X, d)$, we have 


$$
H_{d}\left(\cup_{i \in I} H_{i}, \cup_{i \in I} K_{i}\right) \leq \sup _{i \in I} H_{d}\left(H_{i}, K_{i}\right),
$$

for every $\left(H_{i}\right)_{i \in I}$ and $\left(K_{i}\right)_{i \in I}$ families of elements from $P_{b, c l}(X)$ such that $\cup_{i \in I} H_{i}, \cup_{i \in I} K_{i} \in P_{b, c l}(X)$.

Remark 2.3. The metric spaces $\left(P_{b, c l}(X), H_{d}\right)$ and $\left(P_{c p}(X), H_{d}\right)$ are complete provided that $(X, d)$ is complete.

\section{The shift space}

Let $I$ be a non-empty set.

We denote the set $I^{\mathbb{N}^{*}}$ by $\Lambda(I)$. Thus $\Lambda(I)$ is the set of infinite words with letters from the alphabet $I$ and a standard element $\omega$ of $\Lambda(I)$ can be presented as $\omega=\omega_{1} \omega_{2} \ldots \omega_{n} \omega_{n+1} \ldots$.

We denote the set $I^{\{1,2, \ldots, n\}}$ by $\Lambda_{n}(I)$. Thus $\Lambda_{n}(I)$ is the set of words with letters from the alphabet $I$ of length $n$ and a standard element $\omega$ of $\Lambda_{n}(I)$ can be presented as $\omega=\omega_{1} \omega_{2} \ldots \omega_{n}$. The length of $\omega$ is denoted by $|\omega|$. By $\Lambda_{0}(I)$ we mean the set having only one element, namely the empty word denoted by $\lambda$.

We denote the set $\cup_{n \in \mathbb{N}} \Lambda_{n}(I)$ by $\Lambda^{*}(I)$. Thus $\Lambda^{*}(I)$ is the set of words with letters from the alphabet having finite length.

Given $m, n \in \mathbb{N}$ and two words $\omega=\omega_{1} \omega_{2} \ldots \omega_{n} \in \Lambda_{n}(I)$ and $\theta=\theta_{1} \theta_{2} \ldots \theta_{m} \in$ $\Lambda_{m}(I)$ or $\theta=\theta_{1} \theta_{2} \ldots \theta_{m} \theta_{m+1} \ldots \in \Lambda(I)$, by $\omega \theta$ we mean the concatenation of the words $\omega$ and $\theta$, i.e. $\omega \theta=\omega_{1} \omega_{2} \ldots \omega_{n} \theta_{1} \theta_{2} \ldots \theta_{m}$ and respectively $\omega \theta=\omega_{1} \omega_{2} \ldots \omega_{n} \theta_{1} \theta_{2} \ldots \theta_{m} \theta_{m+1} \ldots$.

For $m \in \mathbb{N}^{*}$ and $\omega=\omega_{1} \omega_{2} \ldots \omega_{n} \omega_{n+1} \ldots \in \Lambda(I)$, by $[\omega]_{m}$ we mean $\omega_{1} \omega_{2} \ldots \omega_{m}$.

For $\omega=\omega_{1} \omega_{2} \ldots \omega_{n} \in \Lambda_{n}(I)$, the word $\theta=\theta_{1} \theta_{2} \ldots \theta_{m} \theta_{m+1} \ldots \in \Lambda(I)$, where $\theta_{n k+i}=\omega_{i}$ for every $k \in \mathbb{N}$ and every $i \in\{1, \ldots, n-1, n\}$, will be denoted by $\dot{\omega}$.

For $i \in I$ one can consider the function $\tau_{i}: \Lambda(I) \rightarrow \Lambda(I)$ given by

$$
\tau_{i}(\omega)=i \omega
$$

for every $\omega \in \Lambda(I)$.

$\Lambda(I)$ becomes a metric space if we endow it with the distance described by $d_{\Lambda}(\omega, \theta)=\left\{\begin{array}{cl}0, & \text { if } \omega=\theta \\ \frac{1}{2^{\min \left\{k \in \mathbb{N}^{*} \mid \omega_{k} \neq \theta_{k}\right\}}}, & \text { if } \omega \neq \theta\end{array}\right.$, where $\omega=\omega_{1} \omega_{2} \omega_{3} \ldots \omega_{n} \omega_{n+1} \ldots$ and $\theta=\theta_{1} \theta_{2} \theta_{3} \ldots \theta_{n}^{2} \theta_{n+1} \ldots$ 


\section{Remarks 2.4.}

a) The convergence in the metric space $\left(\Lambda(I), d_{\Lambda}\right)$ is the convergence on components.

b) $\left(\Lambda(I), d_{\Lambda}\right)$ is a complete metric space.

c) If $I$ is finite, then $\left(\Lambda(I), d_{\Lambda}\right)$ is compact.

Note that points a) and c) follow from the fact that the metric $d_{\Lambda}$ induces the Tychonoff product topology.

Proposition 2.5. Let us consider $\left(\omega_{n}\right) \quad * \subseteq \Lambda(I)$ and $\omega \in \Lambda(I)$ such that $\lim _{n \rightarrow \infty} \omega_{n}=\omega$. Then for every $m \in \mathbb{N}^{*}$ there exists $n_{m} \in \mathbb{N}^{*}$ such that $\left[\omega_{n}\right]_{m} \stackrel{n \rightarrow \infty}{=}=[\omega]_{m}$ for every $n \in \mathbb{N}^{*}, n \geq n_{m}$.

Proof. Ignoring those $\omega_{n}$ which are equal with $\omega$ and supposing that $\omega_{n}=$ $\omega_{n}^{1} \omega_{n}^{2} \ldots \omega_{n}^{k} \ldots$ and $\omega=\omega_{1} \omega_{2} \ldots \omega_{k} \ldots$, for every $m \in \mathbb{N}^{*}$ there exists $n_{m} \in \mathbb{N}^{*}$ such that

$$
\frac{1}{2^{\min \left\{l \in \mathbb{N}^{*} \mid \omega_{n}^{l} \neq \omega_{l}\right\}}}<\frac{1}{2^{m}},
$$

for every $n \in \mathbb{N}^{*}, n \geq n_{m}$. As the previous inequality means that

$$
m<\min \left\{l \in \mathbb{N}^{*} \mid \omega_{n}^{l} \neq \omega_{l}\right\},
$$

we get $\omega_{n}^{1}=\omega_{1}, \omega_{n}^{2}=\omega_{2}, \ldots, \omega_{n}^{m}=\omega_{m}$, i.e

$$
\left[\omega_{n}\right]_{m}=[\omega]_{m},
$$

for every $n \in \mathbb{N}^{*}, n \geq n_{m}$.

\section{Comparison functions and $\varphi$-contractions}

Definition 2.6 (comparison function). A function $\varphi:[0, \infty) \rightarrow[0, \infty)$ is called a comparison function if:

i) $\varphi$ is increasing;

ii) $\varphi(t)<t$ for every $t>0$;

iii) $\varphi$ is right-continuous.

Remark 2.7. If $\varphi:[0, \infty) \rightarrow[0, \infty)$ is a comparison function, then $\lim _{n \rightarrow \infty} \varphi^{[n]}(t)=0$ for every $t \in[0, \infty)$. 
Definition 2.8 ( $\varphi$-contraction). Given a metric space $(X, d)$ and a comparison function $\varphi$, a function $f: X \rightarrow X$ is called $\varphi$-contraction if $d(f(x), f(y)) \leq \varphi(d(x, y))$ for all $x, y \in X$.

A very good reference on comparison functions and $\varphi$-contractions is the survey [10]. See also [8].

\section{Iterated function systems}

Definition 2.9 (iterated function system). A pair $\left((X, d),\left(f_{i}\right)_{i \in I}\right)$ is called an iterated function system (IFS for short) if:

i) $(X, d)$ is a complete metric space;

ii) $I$ is a finite set;

iii) $f_{i}: X \rightarrow X$ is continuous for each $i \in I$;

iv) $f_{i}(B) \in P_{b}(X)$ for every $B \in P_{b}(X)$ and every $i \in I$.

\section{Notations}

1. We shall denote the IFS $\left((X, d),\left(f_{i}\right)_{i \in I}\right)$ by $\mathcal{S}$.

2. In the framework of the above definition, for $\omega=\omega_{1} \omega_{2} \ldots \omega_{n} \in \Lambda_{n}(I)$ and $B$ subset of $X$, by $f_{\omega}(B)$ we mean $\left(f_{\omega_{1}} \circ \ldots \circ f_{\omega_{n}}\right)(B)$.

Definition 2.10 (fractal operator). Given an $\operatorname{IFS} \mathcal{S}=\left((X, d),\left(f_{i}\right)_{i \in I}\right)$, the function $F_{\mathcal{S}}: P_{b, c l}(X) \rightarrow P_{b, c l}(X)$, given by

$$
F_{\mathcal{S}}(B)=\overline{\bigcup_{i \in I} f_{i}(B)},
$$

for every $B \in P_{b, c l}(X)$, is called the fractal operator associated to $\mathcal{S}$.

Definition 2.11 ( $\varphi$-contractive IFS). Given a comparison function $\varphi$, an iterated function system $\mathcal{S}=\left((X, d),\left(f_{i}\right)_{i \in I}\right)$ is called $\varphi$-contractive if $f_{i}$ is a $\varphi$-contraction for each $i \in I$.

Definition 2.12 (point fibred IFS). An iterated function system $\mathcal{S}=$ $\left((X, d),\left(f_{i}\right)_{i \in I}\right)$ is called point fibred if for every $\omega \in \Lambda(I)$ there exists $a_{\omega} \in X$ such that

$$
\lim _{n \rightarrow \infty} f_{[\omega]_{n}}(x)=a_{\omega},
$$

for all $x \in X$.

Definition 2.13 (locally uniformly point fibred IFS). An iterated function system $\mathcal{S}=\left((X, d),\left(f_{i}\right)_{i \in I}\right)$ is called locally uniformly point fibred if it 
is point fibred and for each $x \in X$ there exists an open set $D_{x}$ containing $x$ such that

$$
\lim _{n \rightarrow \infty} \sup _{\omega \in \Lambda(I)} \sup _{y \in D_{x}} d\left(f_{[\omega]_{n}}(y), a_{\omega}\right)=0
$$

Note that the concept of locally uniformly point fibred IFS is the same as the "condition C" that was introduced in Definition 3.1 from [15].

Definition 2.14 (uniformly point fibred IFS). An iterated function system $\mathcal{S}=\left((X, d),\left(f_{i}\right)_{i \in I}\right)$ is called uniformly point fibred if it is point fibred and

$$
\lim _{n \rightarrow \infty} \sup _{\omega \in \Lambda(I)} \sup _{x \in B} d\left(f_{[\omega]_{n}}(x), a_{\omega}\right)=0,
$$

for every $B \in P_{b, c l}(X)$.

Definition 2.15 (IFS having attractor). We say that an iterated function system $\mathcal{S}=\left((X, d),\left(f_{i}\right)_{i \in I}\right)$ has an attractor if there exists $A_{\mathcal{S}} \in P_{b, c l}(X)$ such that:

i)

$$
F_{\mathcal{S}}\left(A_{\mathcal{S}}\right)=A_{\mathcal{S}}
$$

ii)

$$
\lim _{n \rightarrow \infty} H_{d}\left(F_{\mathcal{S}}^{[n]}(B), A_{\mathcal{S}}\right)=0
$$

for each $B \in P_{b, c l}(X)$.

Remark 2.16. $F_{\mathcal{S}}$ has a unique fixed point, namely $A_{\mathcal{S}} \in P_{c p}(X)$, which is called the attractor of $\mathcal{S}$.

Indeed, if for some $B \in P_{b, c l}(X)$ we have $F_{\mathcal{S}}(B)=B$, then

$$
0 \stackrel{\mathrm{ii})}{=} \lim _{n \rightarrow \infty} H_{d}\left(F_{\mathcal{S}}^{[n]}(B), A_{\mathcal{S}}\right)=\lim _{n \rightarrow \infty} H_{d}\left(B, A_{\mathcal{S}}\right)=H_{d}\left(B, A_{\mathcal{S}}\right),
$$

so $B=A_{\mathcal{S}}$. In addition, $\lim _{n \rightarrow \infty} H_{d}\left(F_{\mathcal{S}}^{[n]}(K), A_{\mathcal{S}}\right)=0$ for every $K \in P_{c p}(X) \subseteq$ $P_{b, c l}(X)$ and in view of Proposition 2.7 from [17] we conclude that $A_{\mathcal{S}} \in$ $P_{c p}(X)$.

Definition 2.17 (diameter diminishing to zero IFS). An iterated function system $\mathcal{S}=\left((X, d),\left(f_{i}\right)_{i \in I}\right)$ is called diameter diminishing to zero iterated 
function systems if for every $B \in P_{b, c l}(X)$ there exists $M_{B} \in P_{b, c l}(X)$ such that:

i)

$$
B \subseteq M_{B}
$$

ii)

$$
F_{\mathcal{S}}\left(M_{B}\right) \subseteq M_{B}
$$

iii)

$$
\lim _{n \rightarrow \infty} \max _{\omega \in \Lambda_{n}(I)} \operatorname{diam}\left(f_{\omega}\left(M_{B}\right)\right)=0 .
$$

Definition 2.18 (hyperbolic $\varphi$-contractive IFS). Given a comparison function $\varphi$, an iterated function system $\mathcal{S}=\left((X, d),\left(f_{i}\right)_{i \in I}\right)$ is called hyperbolic $\varphi$-contractive if there exists a distance $d_{1}$ on $X$ such that:

i) $d$ and $d_{1}$ are topologically equivalent;

ii) $\left(X, d_{1}\right)$ is complete;

iii) $\mathcal{S}_{1}=\left(\left(X, d_{1}\right),\left(f_{i}\right)_{i \in I}\right)$ is $\varphi$-contractive.

\section{Remarks 2.19.}

a) The concepts of hyperbolic locally uniformly point fibred IFS, hyperbolic uniformly point fibred IFS, IFS having hyperbolic attractor and hyperbolic diameter diminishing to zero IFS could be defined having as model Definition 2.18.

b) An iterated function system $\mathcal{S}$ which is uniformly point fibred is locally uniformly point fibred.

c) An iterated function system $\mathcal{S}$ which is hyperbolic uniformly point fibred is hyperbolic locally uniformly point fibred.

d) As one of the referees of this paper noted, Definition 2.15 raises the question whether there exists and IFS with hyperbolic attractor and not having attractor.

\section{THE PROPERTIES OF DIAMETER DIMINISHING TO} ZERO ITERATED FUNCTION SYSTEMS

Proposition 3.1. Given a diameter diminishing to zero IFS $\mathcal{S}=\left((X, d),\left(f_{i}\right)_{i \in I}\right)$, for every $\omega \in \Lambda(I)$ there exists $a_{\omega} \in X$ such that

$$
\bigcap_{n \in \mathbb{N}^{*}} \overline{f_{[\omega]_{n}}(B)}=\left\{a_{\omega}\right\},
$$


for every $B \in P_{b, c l}(X)$ having the property that $F_{\mathcal{S}}(B) \subseteq B$.

Proof. Let $\omega \in \Lambda(I)$ be fixed.

Claim 1. For every $B \in P_{b, c l}(X)$ such that $F_{\mathcal{S}}(B) \subseteq B$ there exists $a_{\omega, B} \in X$ such that

$$
\bigcap_{n \in \mathbb{N}^{*}} \overline{f_{[\omega]_{n}}(B)}=\left\{a_{\omega, B}\right\} .
$$

Justification of Claim 1. As $f_{i}(B) \subseteq B$ for each $i \in I$, we have

$$
f_{[\omega]_{n+1}}(B) \subseteq f_{[\omega]_{n}}(B),
$$

for every $n \in \mathbb{N}^{*}$. Since

$$
\begin{gathered}
\operatorname{diam}\left(\overline{f_{[\omega]_{n}}(B)}\right)=\operatorname{diam}\left(f_{[\omega]_{n}}(B)\right) \leq \\
\leq \max _{\theta \in \Lambda_{n}(I)} \operatorname{diam}\left(f_{\theta}(B)\right) \stackrel{B \subseteq M_{B}}{\leq} \max _{\theta \in \Lambda_{n}(I)} \operatorname{diam}\left(f_{\theta}\left(M_{B}\right)\right),
\end{gathered}
$$

for every $n \in \mathbb{N}^{*}$, where $M_{B}$ is the element of $P_{b, c l}(X)$ whose existence is stated in Definition 2.17. Using iii) of the same Definition, via (1), we conclude that

$$
\lim _{n \rightarrow \infty} \operatorname{diam}\left(\overline{f_{[\omega]_{n}}(B)}\right)=0 .
$$

Therefore, according to Cantor's theorem, there exists $a_{\omega, B} \in X$ such that

$$
\bigcap_{n \in \mathbb{N}^{*}} \overline{f_{[\omega]_{n}}(B)}=\left\{a_{\omega, B}\right\} .
$$

The justification of the claim is done.

Claim 2. For every $B, C \in P_{b, c l}(X)$ such that $F_{\mathcal{S}}(B) \subseteq B, F_{\mathcal{S}}(C) \subseteq C$ and $B \subseteq C$, we have

$$
a_{\omega, B}=a_{\omega, C} .
$$

Justification of Claim 2. Indeed

$$
\left\{a_{\omega, B}\right\} \stackrel{\text { Claim } 1}{=} \underset{n \in \mathbb{N}^{*}}{\bar{f}_{[\omega]_{n}}(B)} \subseteq \underset{n \in \mathbb{N}^{*}}{f_{[\omega]_{n}}(C)} \stackrel{\text { Claim } 1}{=}\left\{a_{\omega, C}\right\}
$$

and the justification of the claim is done.

Claim 3. For every $B, C \in P_{b, c l}(X)$ such that $F_{\mathcal{S}}(B) \subseteq B$ and $F_{\mathcal{S}}(C) \subseteq$ $C$, we have

$$
a_{\omega, B}=a_{\omega, C}
$$


Justification of Claim 3. Since

$$
B \cup C \in P_{c l, b}(X)
$$

and

$$
F_{\mathcal{S}}(B \cup C) \subseteq F_{\mathcal{S}}(B) \cup F_{\mathcal{S}}(C) \subseteq B \cup C
$$

we infer that

$$
a_{\omega, B} \stackrel{\text { Claim } 2}{=} a_{\omega, B \cup C} \stackrel{\text { Claim } 2}{=} a_{\omega, C},
$$

and the justification of the claim is done.

Hence $\left\{a_{\omega, B} \mid B \in P_{b, c l}(X)\right.$ such that $\left.F_{\mathcal{S}}(B) \subseteq B\right\}$ has only one element, which is denoted by $a_{\omega}$.

In view of the previous Proposition, given a diameter diminishing to zero IFS $\mathcal{S}=\left((X, d),\left(f_{i}\right)_{i \in I}\right)$ one can consider the function $\pi: \Lambda(I) \rightarrow X$ given by

$$
\pi(\omega)=a_{\omega}
$$

for each $\omega \in \Lambda(I)$.

Proposition 3.2. Each diameter diminishing to zero IFS is uniformly point fibred.

Proof. If $\mathcal{S}=\left((X, d),\left(f_{i}\right)_{i \in I}\right)$, then, according to Definition 2.17, for each $B \in P_{b, c l}(X)$ exists $M_{B} \in P_{b, c l}(X)$ such that $B \subseteq M_{B}$ and $F_{\mathcal{S}}\left(M_{B}\right) \subseteq M_{B}$. Consequently, for every $\omega \in \Lambda(I)$, we have

$$
\begin{gathered}
\sup _{x \in B} d\left(f_{[\omega]_{n}}(x), a_{\omega}\right) \leq \sup _{x \in M_{B}} d\left(f_{[\omega]_{n}}(x), a_{\omega}\right) \stackrel{a_{\omega} \in \overline{f_{[\omega]_{n}}\left(M_{B}\right)}}{\leq} \\
\leq \operatorname{diam}\left(\overline{f_{[\omega]_{n}}\left(M_{B}\right)}\right) \leq \max _{\theta \in \Lambda_{n}(I)} \operatorname{diam}\left(f_{\theta}\left(M_{B}\right)\right)
\end{gathered}
$$

for every $n \in \mathbb{N}^{*}$. Hence

$$
\sup _{\omega \in \Lambda(I) x \in B} \sup _{\omega} d\left(f_{[\omega]_{n}}(x), a_{\omega}\right) \leq \max _{\omega \in \Lambda_{n}(I)} \operatorname{diam}\left(f_{\omega}\left(M_{B}\right)\right),
$$

for every $n \in \mathbb{N}^{*}$, so, in view of Definition 2.17, we obtain

$$
\lim _{n \rightarrow \infty} \sup _{\omega \in \Lambda(I) x \in B} \sup _{d} d\left(f_{[\omega]_{n}}(x), a_{\omega}\right)=0,
$$

i.e. $\mathcal{S}$ is uniformly point fibred. 
Proposition 3.3. For each diameter diminishing to zero IFS $\mathcal{S}=$ $\left((X, d),\left(f_{i}\right)_{i \in I}\right)$, we have

$$
f_{i} \circ \pi=\pi \circ \tau_{i}
$$

for every $i \in I$.

Proof. For a fixed $B \in P_{b, c l}(X)$ such that $F_{\mathcal{S}}(B) \subseteq B$, we have

$$
\begin{aligned}
\left\{f_{i}(\pi(\omega))\right\} & =\left\{f_{i}\left(a_{\omega}\right)\right\}=f_{i}\left(\underset{n \in \mathbb{N}^{*}}{\cap} \overline{f_{[\omega]_{n}}(B)}\right) \stackrel{f_{i} \text { continuous }}{\subseteq} \underset{n \in \mathbb{N}^{*}}{\subseteq} \overline{f_{i}\left(f_{[\omega]_{n}}(B)\right)}= \\
& =\bigcap_{n \in \mathbb{N}^{*}} \overline{f_{[i \omega]_{n}}(B)}=\left\{a_{i \omega}\right\}=\{\pi(i \omega)\}=\left\{\pi\left(\tau_{i}(\omega)\right)\right\},
\end{aligned}
$$

i.e.

$$
\left(f_{i} \circ \pi\right)(\omega)=\left(\pi \circ \tau_{i}\right)(\omega),
$$

for every $\omega \in \Lambda(I)$ and every $i \in I$.

Proposition 3.4. For each diameter diminishing to zero IFS $\mathcal{S}=$ $\left((X, d),\left(f_{i}\right)_{i \in I}\right)$, the function $\pi$ is continuous.

Proof. First of all, let us chose a fixed $B \in P_{b, c l}(X)$ such that $F_{\mathcal{S}}(B) \subseteq B$. Now let us consider $\omega \in \Lambda(I)$ and a sequence $\left(\omega_{n}\right)_{n \in \mathbb{N}^{*}}$ of elements of $\Lambda(I)$ converging to $\omega$. Therefore, in view of Proposition 2.5, for each $m \in \mathbb{N}^{*}$ there exists $n_{m} \in \mathbb{N}^{*}$ such that

$$
\left[\omega_{n}\right]_{m}=[\omega]_{m},
$$

for every $n \in \mathbb{N}^{*}, n \geq n_{m}$. Let us consider a fixed, but arbitrarily chosen $\varepsilon>0$. Taking into account Definition 2.17, there exists $m_{\varepsilon} \in \mathbb{N}^{*}$ such that

$$
\max _{\theta \in \Lambda_{m_{\varepsilon}}(I)} \operatorname{diam}\left(f_{\theta}(B)\right)<\varepsilon .
$$

Then for every $n \in \mathbb{N}^{*}, n \geq n_{m_{\varepsilon}}$, as $\pi\left(\omega_{n}\right) \in \overline{f_{\left[\omega_{n}\right]_{m_{\varepsilon}}}(B)}$ and $\pi(\omega) \in \overline{f_{[\omega]_{m_{\varepsilon}}}(B)}$, with the notation $\left[\omega_{n}\right]_{m_{\varepsilon}} \stackrel{(2)}{=}[\omega]_{m_{\varepsilon}} \stackrel{\text { not }}{=} \beta_{m_{\varepsilon}} \in \Lambda_{m_{\varepsilon}}(I)$, we get

$$
d\left(\pi\left(\omega_{n}\right), \pi(\omega)\right) \leq \operatorname{diam}\left(\overline{f_{\beta_{m_{\varepsilon}}}(B)}\right)=\operatorname{diam}\left(f_{\beta_{m_{\varepsilon}}}(B)\right) \stackrel{(3)}{<} \varepsilon .
$$

The last relation assures us that sequence $\left(\pi\left(\omega_{n}\right)\right)_{n \in \mathbb{N}^{*}}$ converges to $\pi(\omega)$ and this shows that $\pi$ is continuous.

Proposition 3.5. Each diameter diminishing to zero IFS $\mathcal{S}=\left((X, d),\left(f_{i}\right)_{i \in I}\right)$ has attractor and $A_{\mathcal{S}}=\pi(\Lambda(I))=\left\{a_{\omega} \mid \omega \in \Lambda(I)\right\}$. 
Proof. Let us note that:

i)

$$
\pi(\Lambda(I)) \stackrel{\text { Proposition 3.4 \& Remark 2.4, c) }}{\in} P_{c p}(X) \subseteq P_{b, c l}(X) ;
$$

ii)

$$
\begin{gathered}
F_{\mathcal{S}}(\pi(\Lambda(I))) \stackrel{\text { Definition } 2.10}{=} \cup_{i \in I} f_{i}(\pi(\Lambda(I))) \stackrel{\text { Proposition 3.3 }}{=} \\
=\cup_{i \in I} \pi\left(\tau_{i}(\Lambda(I))\right)=\pi\left(\cup_{i \in I} \tau_{i}(\Lambda(I))\right)=\pi(\Lambda(I)) .
\end{gathered}
$$

Moreover,

$$
\lim _{n \rightarrow \infty} H_{d}\left(F_{\mathcal{S}}^{[n]}(B), \pi(\Lambda(I))\right)=0,
$$

for each $B \in P_{b, c l}(X)$.

Indeed, let us consider a fixed, but arbitrarily chosen $B \in P_{b, c l}(X)$. We have

$$
\begin{aligned}
& H_{d}\left(F_{\mathcal{S}}^{[n]}(B), \pi(\Lambda(I))\right)=H_{d}\left(\underset{\omega \in \Lambda(I)}{\cup} f_{[\omega]_{n}}(B), \underset{\omega \in \Lambda(I)}{\bigcup}\left\{a_{\omega}\right\}\right) \stackrel{\text { Proposition } 2.2}{\leq} \\
& \leq \sup _{\omega \in \Lambda(I)} H_{d}\left(f_{[\omega]_{n}}(B),\left\{a_{\omega}\right\}\right)=\sup _{\omega \in \Lambda(I)} \max \left\{\sup _{x \in B} d\left(f_{[\omega]_{n}}(x), a_{\omega}\right), \inf _{x \in B} d\left(f_{[\omega]_{n}}(x), a_{\omega}\right)\right\}= \\
& a_{\omega} \in \overline{\frac{f_{[\omega]_{n}}(B)}{=}} \sup _{\omega \in \Lambda(I) x \in B} \sup _{x \in B} d\left(f_{[\omega]_{n}}(x), a_{\omega}\right),
\end{aligned}
$$

for every $n \in \mathbb{N}^{*}$ and taking into account Proposition 3.2, we conclude that $\lim _{n \rightarrow \infty} H_{d}\left(F_{\mathcal{S}}^{[n]}(B), \pi(\Lambda(I))\right)=0$. Consequently $\pi(\Lambda(I))$ is the attractor of $\mathcal{S}$.

Proposition 3.6. For each diameter diminishing to zero IFS $\mathcal{S}=$ $\left((X, d),\left(f_{i}\right)_{i \in I}\right)$, we have

$$
\left\{a_{\dot{\omega}}\right\}=F i x\left(f_{\omega}\right)
$$

for each $\omega \in \Lambda^{*}(I) \backslash\{\lambda\}$.

Proof. Since

$$
f_{\omega}\left(a_{\dot{\omega}}\right)=f_{\omega}(\pi(\dot{\omega})) \stackrel{\text { Proposition } 3.3}{=} \pi(\omega \dot{\omega})=\pi(\dot{\omega})=a_{\dot{\omega}},
$$

we conclude that

$$
\left\{a_{\dot{\omega}}\right\} \subseteq \operatorname{Fix}\left(f_{\omega}\right)
$$

for every $\omega \in \Lambda^{*}(I) \backslash\{\lambda\}$. 
Now we prove that

$$
\operatorname{Fix}\left(f_{\omega}\right) \subseteq\left\{a_{\dot{\omega}}\right\},
$$

for every $\omega \in \Lambda^{*}(I) \backslash\{\lambda\}$.

Let us consider $z \in F i x\left(f_{\omega}\right)$ (so $z \in \underset{n \in \mathbb{N}^{*}}{ }\left\{f_{[\dot{\omega}]_{n|\omega|}}(z)\right\}$ ). Then, in view of Definition 2.17, there exists $M_{\{z\}} \in P_{b, c l}(X)$ such that $z \in M_{\{z\}}$ and $F_{\mathcal{S}}\left(M_{\{z\}}\right) \subseteq M_{\{z\}}$. Hence

$$
f_{i}\left(M_{\{z\}}\right) \subseteq F_{\mathcal{S}}\left(M_{\{z\}}\right) \subseteq M_{\{z\}},
$$

for every $i \in I$, so $\left(\overline{f_{[\dot{\omega}]_{n|\omega|}}\left(M_{\{z\}}\right)}\right)_{n \in \mathbb{N}^{*}}$ is a decreasing sequence and therefore

$$
\bigcap_{n \in \mathbb{N}^{*}} \overline{f_{[\dot{\omega}]_{n|\omega|}}\left(M_{\{z\}}\right)}=\bigcap_{n \in \mathbb{N}^{*}} \overline{f_{[\dot{\omega}]_{n}}\left(M_{\{z\}}\right)} \stackrel{\text { Proposition }}{=}\left\{.1\left\{a_{\dot{\omega}}\right\} .\right.
$$

Consequently

$$
z \in \bigcap_{n \in \mathbb{N}}\left\{f_{[\dot{\omega}]_{n|\omega|}}(z)\right\} \subseteq \bigcap_{n \in \mathbb{N}} f_{[\dot{\omega}]_{n|\omega|}}\left(M_{\{z\}}\right) \subseteq \bigcap_{n \in \mathbb{N}} \overline{f_{[\dot{\omega}]_{n|\omega|}}\left(M_{\{z\}}\right)}=\left\{a_{\dot{\omega}}\right\}
$$

and (4) is justified.

The relations (4) and (5) assure us that Fix $\left(f_{\omega}\right)=\left\{a_{\dot{\omega}}\right\}$.

Proposition 3.7. For each diameter diminishing to zero IFS $\mathcal{S}=$ $\left((X, d),\left(f_{i}\right)_{i \in I}\right)$, we have

$$
A_{\mathcal{S}}=\overline{\left\{a_{\dot{\omega}} \mid \omega \in \Lambda^{*}(I) \backslash\{\lambda\}\right.} .
$$

Proof. As $\left\{a_{\dot{\omega}} \mid \omega \in \Lambda^{*}(I) \backslash\{\lambda\}\right\} \subseteq A_{\mathcal{S}}$ and $A_{\mathcal{S}}$ is compact, it suffices to prove that

$$
A_{\mathcal{S}} \subseteq \overline{\left\{a_{\dot{\omega}} \mid \omega \in \Lambda^{*}(I) \backslash\{\lambda\}\right\}} .
$$

To this aim, let us consider $x \in A_{\mathcal{S}}$. For an arbitrary neighborhood $V$ of $x$, there exists an open subset $D$ of $X$ such that $x \in D \subseteq V$. As $A_{\mathcal{S}}=\left\{a_{\omega} \mid \omega \in \Lambda(I)\right\}$, there exists $\omega_{x} \in \Lambda(I)$ such that $x=a_{\omega_{x}}$. Hence

$$
\bigcap_{n \in \mathbb{N}^{*}} f_{\left[\omega_{x}\right]_{n}}\left(A_{\mathcal{S}}\right) \stackrel{\text { Proposition } 3.1}{=}\left\{a_{\omega_{x}}\right\} \subseteq D
$$

and since the sequence $\left(f_{\left[\omega_{x}\right]_{n}}\left(A_{\mathcal{S}}\right)\right)_{n \in \mathbb{N}^{*}} \subseteq P_{c p}(X)$ is decreasing and $D$ is open, there exists $m \in \mathbb{N}$ such that

$$
f_{\left[\omega_{x}\right]_{m}}\left(A_{\mathcal{S}}\right) \subseteq D
$$


(see, for example, Corollary 3.1.5 from [7]). Therefore

$$
a_{\left[\omega_{x}\right]_{m}} \stackrel{\text { Proposition } 3.6}{=} f_{\left[\omega_{x}\right]_{m}}\left(a_{\left[\omega_{x}\right]_{m}}\right) \in f_{\left[\omega_{x}\right]_{m}}\left(A_{\mathcal{S}}\right) \stackrel{(6)}{\subseteq} D \subseteq V
$$

SO

$$
V \cap\left\{a_{\dot{\omega}} \mid \omega \in \Lambda^{*}(I) \backslash\{\lambda\}\right\} \neq \emptyset .
$$

Hence $x \in \overline{\left\{a_{\dot{\omega}} \mid \omega \in \Lambda^{*}(I) \backslash\{\lambda\}\right\}}$ and the proof is done.

\section{THE MAIN RESULT}

Theorem 4.1. For an iterated function system $\mathcal{S}=\left((X, d),\left(f_{i}\right)_{i \in I}\right)$, the following statements are equivalent:

1. There exists a comparison function $\varphi$ such that $\mathcal{S}$ is hyperbolic $\varphi$ contractive.

2. $\mathcal{S}$ is hyperbolic locally uniformly point fibred.

3. $\mathcal{S}$ is hyperbolic uniformly point fibred.

4. $\mathcal{S}$ is a hyperbolic diameter diminishing to zero iterated function system.

5. $\mathcal{S}$ has hyperbolic attractor and there exists a continuous surjection $\pi: \Lambda(I) \rightarrow A_{\mathcal{S}}$ such that

$$
f_{i} \circ \pi=\pi \circ \tau_{i}
$$

for all $i \in I$.

Proof.

The argument used for the justification of Remark 3.1 from [15] ensures the validity of 1$) \Rightarrow 3$ ).

For 3$) \Rightarrow 2$ ) see Remark 2.19, c).

For 2$) \Rightarrow 1$ ) see Theorem 3.1 from [15].

$1) \Rightarrow 4)$ First of all, note that for every $B \in P_{b, c l}(X)$, we have

$$
\max _{\omega \in \Lambda_{n}(I)} \operatorname{diam}\left(f_{\omega}(B)\right) \leq \varphi^{[n]}(\operatorname{diam}(B))
$$

for every $n \in \mathbb{N}^{*}$, so, taking into account Remark 2.7, we obtain that

$$
\lim _{n \rightarrow \infty} \max _{\omega \in \Lambda_{n}(I)} \operatorname{diam}\left(f_{\omega}(B)\right)=0 .
$$

In addition, since there exists a unique $A_{\mathcal{S}} \in P_{b, c l}(X)$ such that $F_{\mathcal{S}}\left(A_{\mathcal{S}}\right)=$ $A_{\mathcal{S}}$ and $\lim _{n \rightarrow \infty} F_{\mathcal{S}}^{[n]}(B)=A_{\mathcal{S}}$ for every $B \in P_{b, c l}(X)$ (see Theorem 2.5 from [5]), one can consider the set

$$
M_{B}=\overline{A_{\mathcal{S}} \cup \cup_{n \in \mathbb{N}} F_{S}^{[n]}(B)} \supseteq B .
$$


Note that as $\lim _{n \rightarrow \infty} F_{S}^{[n]}(B)=A_{\mathcal{S}}$, there exists $n_{1} \in \mathbb{N}$ and $\varepsilon>0$ such that $F_{S}^{[n]}(B) \subseteq E_{\varepsilon}\left(A_{\mathcal{S}}\right)$ for each $n \in \mathbb{N}, n \geq n_{1}$. Hence $\underset{n \in \mathbb{N}, n \geq n_{1}}{\cup} F_{S}^{[n]}(B) \in$ $P_{b}(X)$, so, via Definition 2.9, iv), we infer that $A_{\mathcal{S}} \cup \underset{n \in \mathbb{N}}{\cup} F_{S}^{[n]}(B) \in P_{b}(X)$. Consequently

$$
M_{B} \in P_{b, c l}(X) .
$$

Moreover, we have

$$
\begin{aligned}
& F_{S}\left(M_{B}\right)=F_{S}\left(\overline{A_{\mathcal{S}} \cup \cup_{n \in \mathbb{N}} F_{S}^{[n]}(B)}\right)=\overline{\cup_{i \in I} f_{i}\left(\overline{A_{\mathcal{S}} \cup \cup_{n \in \mathbb{N}} F_{S}^{[n]}(B)}\right)} \subseteq
\end{aligned}
$$

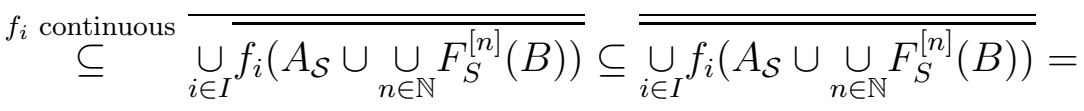

$$
\begin{aligned}
& =\overline{F_{S}\left(A_{\mathcal{S}} \cup \cup_{n \in \mathbb{N}} F_{S}^{[n]}(B)\right)} \subseteq \overline{\left.F_{S}\left(A_{\mathcal{S}}\right) \cup \cup_{n \in \mathbb{N}} F_{S}\left(F_{S}^{[n]}(B)\right)\right)} \subseteq \\
& \subseteq \overline{\left.A_{\mathcal{S}} \cup \cup_{n \in \mathbb{N}} F_{S}^{[n+1]}(B)\right)} \subseteq M_{B} .
\end{aligned}
$$

Therefore, in view of (7), (8), (9) and (10), $\mathcal{S}$ is a hyperbolic diameter diminishing to zero iterated function system.

$4) \Rightarrow 5$ ) See the results from Section 3 .

$5) \Rightarrow 2$ ) Let $d_{1}$ be the distance on $X$ whose existence is assured by Remarks 2.19, a).

First of all let us note that $A_{\mathcal{S}} \in P_{c p}(X)$ (since $A_{\mathcal{S}}=\pi(\Lambda(I)), \pi$ is continuous and $\Lambda(I)$ compact -see Remark 2.4, c)-).

Claim 1.

$$
\lim _{n \rightarrow \infty} \max _{\omega \in \Lambda_{n}(I)} \operatorname{diam}\left(f_{\omega}\left(A_{\mathcal{S}}\right)\right)=0 .
$$

Justification of Claim 1. See Lemma 1.6 from [11].

Claim 2. For every $\omega \in \Lambda(I)$, the set $\bigcap_{n \in \mathbb{N}^{*}} f_{[\omega]_{n}}\left(A_{\mathcal{S}}\right)$ consists on only one element denoted by $b_{\omega}$.

Justification of Claim 2. Just use Cantor's theorem and Claim 1.

Let us consider $\varepsilon>0$ fixed, but arbitrarily chosen.

Then, via Claim 1, there exists $n_{0} \in \mathbb{N}$ such that

$$
\operatorname{diam}\left(f_{\alpha}\left(A_{\mathcal{S}}\right)\right)<\frac{\varepsilon}{3}
$$

for every $\alpha \in \Lambda_{n_{0}}(I)$. 
For every $\alpha \in \Lambda_{n_{0}}(I)$ there exists $\delta_{\alpha}>0$ such that

$$
f_{\alpha}\left(E_{\delta_{\alpha}}\left(A_{\mathcal{S}}\right)\right) \subseteq E_{\frac{\varepsilon}{3}}\left(f_{\alpha}\left(A_{\mathcal{S}}\right)\right) .
$$

Indeed, the continuity of $f_{\alpha}$ assures us that for every $x \in A_{\mathcal{S}}$ there exists $\delta_{\alpha, x}>0$ such that $f_{\alpha}\left(B\left(x, 2 \delta_{\alpha, x}\right)\right) \subseteq B\left(f_{\alpha}(x), \frac{\varepsilon}{3}\right)$. In view of the compactness of $A_{\mathcal{S}}$ there exist $p \in \mathbb{N}^{*}$ and $x_{1}, \ldots, x_{p} \in A_{\mathcal{S}}$ such that $A_{\mathcal{S}} \subseteq \bigcup_{j=1}^{p} B\left(x_{j}, \delta_{\alpha, x_{j}}\right)$. Let us consider $\delta_{\alpha}=\min \left\{\delta_{\alpha, x_{1}}, \ldots, \delta_{\alpha, x_{p}}\right\}>0$. If $u \in E_{\delta_{\alpha}}\left(A_{\mathcal{S}}\right)$, there exists $v \in A_{\mathcal{S}}$ such that $d_{1}(u, v)<\delta_{\alpha}$. In addition, there exists $j_{v} \in\{1, \ldots, p\}$ having the property that $d_{1}\left(x_{j_{v}}, v\right)<\delta_{\alpha, x_{j_{v}}}$. Consequently we have $d_{1}\left(u, x_{j_{v}}\right) \leq$ $d_{1}(u, v)+d_{1}\left(v, x_{j_{v}}\right)<\delta_{\alpha}+\delta_{\alpha, x_{j v}}<2 \delta_{\alpha, x_{j v}}$, so $f_{\alpha}(u) \in f_{\alpha}\left(B\left(x_{j_{v}}, 2 \delta_{\alpha, x_{j v}}\right)\right) \subseteq$ $B\left(f_{\alpha}\left(x_{j_{v}}\right), \frac{\varepsilon}{3}\right)$, i.e. $f_{\alpha}(u) \in E_{\frac{\varepsilon}{3}}\left(f_{\alpha}\left(A_{\mathcal{S}}\right)\right)$. As $u$ was arbitrarily chosen in $A_{\mathcal{S}}$, the justification of (12) is done.

Let us consider

$$
\delta=\min \left\{\delta_{\alpha} \mid \alpha \in \Lambda_{n_{0}}(I)\right\} .
$$

Since $\lim _{n \rightarrow \infty} F_{\mathcal{S}}^{[n]}(\overline{B(x, \eta)})=A_{\mathcal{S}}$ for every $x \in X$ and $\eta>0$, there exists $m_{0} \in \mathbb{N}^{*}$ such that

$$
F_{S}^{[m]}(\overline{B(x, \eta)}) \subseteq E_{\delta}\left(A_{\mathcal{S}}\right)
$$

for every $m \in \mathbb{N}^{*}, m \geq m_{0}$. For an arbitrary $\omega \in \Lambda(I)$ let us consider $[\omega]_{n_{0}} \stackrel{\text { not }}{=} \alpha$. Then, for $m \in \mathbb{N}, m \geq m_{0}$, if $[\omega]_{n_{0}+m}=\alpha \beta$, where $\beta \in \Lambda_{m}(I)$, via Claim 2, we have

$$
\begin{gathered}
d_{1}\left(f_{[\omega]_{n_{0}+m}}(y), b_{\omega}\right)=d_{1}\left(f_{\alpha}\left(f_{\beta}(y)\right), b_{\omega}\right) \leq \sup _{u \in F_{S}^{m}(\overline{B(x, \eta))}} d_{1}\left(f_{\alpha}(u), b_{\omega}\right) \leq \\
\stackrel{(13)}{\leq} \sup _{u \in E_{\delta}\left(A_{\mathcal{S}}\right)} d_{1}\left(f_{\alpha}(u), b_{\omega}\right) \stackrel{(12)}{\leq} \sup _{\left.v \in E_{\frac{\varepsilon}{3}}\left(f_{\alpha}\left(A_{\mathcal{S}}\right)\right)\right)} d_{1}\left(v, b_{\omega}\right) \leq \\
\leq \operatorname{diam}\left(E_{\frac{\varepsilon}{3}}\left(f_{\alpha}\left(A_{\mathcal{S}}\right)\right)\right) \leq \frac{2 \varepsilon}{3}+\operatorname{diam}\left(f_{\alpha}\left(A_{\mathcal{S}}\right)\right) \stackrel{(11)}{<} \frac{2 \varepsilon}{3}+\frac{\varepsilon}{3}=\varepsilon,
\end{gathered}
$$

for every $y \in \overline{B(x, \eta)}$, so

$$
\sup _{\omega \in \Lambda(I)} \sup _{y \in \overline{B(x, \eta)}} d_{1}\left(f_{[\omega]_{n_{0}+m}}(y), b_{\omega}\right) \leq \varepsilon,
$$

for every $m \in \mathbb{N}, m \geq m_{0}$. Hence

$$
\lim _{n \rightarrow \infty} \sup _{\omega \in \Lambda(I)} \sup _{y \in \overline{B(x, \eta)}} d_{1}\left(f_{[\omega]_{n}}(y), b_{\omega}\right)=0,
$$


i.e. $\mathcal{S}$ is hyperbolic uniformly point fibred.

\section{FINAL REMARKS}

A. Let us recall the concept of topologically contractive iterated function system that was introduced by A. Mihail (see [19]) and by A. Tetenov (see [20] and [21]) under a different name, namely self-similar topological structure satisfying condition $(\mathrm{P})$. It is part of the last decades trend to establish purely topological conditions on an iterated function system in order to guarantee the existence of attractors.

Definition 5.1. A pair $\left((X, \tau),\left(f_{i}\right)_{i \in I}\right)$ is called a topologically contractive iterated function system if:

i) $(X, \tau)$ is a topological space;

ii) $I$ is a finite set;

iii) $f_{i}: X \rightarrow X$ is continuous for every $i \in I$;

iv) for every $K \in P_{c p}(X)$ there exists $C_{K} \in P_{c p}(X)$ such that $K \subseteq C_{K}$ and $\cup_{i \in I} f_{i}\left(C_{K}\right) \subseteq C_{K}$;

v) for every $C \in P_{c p}(X)$ such that $\cup_{i \in I} f_{i}(C) \subseteq C$ and every $\omega \in \Lambda(I)$, the set $\bigcap_{n \in \mathbb{N}^{*}} f_{[\omega]_{n}}(C)$ is a singleton.

Particular cases of the above mentioned concept were considered by A. Edalat (see [6]) under de name of weakly hyperbolic iterated function systems and by B. Kieninger (see [12]) under the name of point fibred iterated function systems.

A comparison between conditions i) and ii) from Definition 2.17 and condition iv) from Definition 5.1 and between the conclusion of Proposition 3.1 and condition v) from Definition 5.1 shows that the concept of diameter diminishing to zero iterated function systems is a counterpart in terms of metric spaces of the one of topologically contractive iterated function system. Note that it is dealing with closed and bounded (not necessarly compact) sets.

B. Even though Propositions 3.3, 3.4 and 3.5 follow from Proposition 3.2 and Theorem 3.1 from [15] we presented their proofs as they are elementary, while Theorem 3.1 from [15] is complicated and nontrivial.

C. For an iterated function system $\mathcal{S}=\left(\left((X, d),\left(f_{i}\right)_{i \in I}\right)\right)$ we can consider the following conditions: 
$\left.1^{\prime}\right)$ There exists a comparison function $\varphi$ such that $\mathcal{S}$ is $\varphi$-contractive.

$\left.2^{\prime}\right) \mathcal{S}$ is locally uniformly point fibred.

$\left.3^{\prime}\right) \mathcal{S}$ is uniformly point fibred.

$\left.4^{\prime}\right) \mathcal{S}$ is diameter diminishing to zero iterated function system.

$\left.5^{\prime}\right) \mathcal{S}$ has an attractor and there exists a continuous function $\pi: \Lambda(I) \rightarrow$ $A_{\mathcal{S}}$ such that $\pi \circ \tau_{i}=f_{i} \circ \pi$ for all $i \in I$.

Actually the proof of Theorem 4.1 ensures the validity of the following implications: $\left.\left.\left.\left.\left.\left.\left.1^{\prime}\right) \rightarrow 4^{\prime}\right), 4^{\prime}\right) \rightarrow 3^{\prime}\right), 3^{\prime}\right) \rightarrow 2^{\prime}\right), 4^{\prime}\right) \rightarrow 5^{\prime}$ ) and $\left.5^{\prime}\right) \rightarrow 2^{\prime}$ ).

We raise the following question: is $\left.2^{\prime}\right) \rightarrow 4^{\prime}$ ) valid?

If this is true, then we get the equivalence of $\left.2^{\prime}\right), 3^{\prime}$ ), $4^{\prime}$ ) and $5^{\prime}$ ).

Acknowledgement. We want to thank the referees whose generous and valuable remarks brought improvements to the paper (specially by adding Section 5) and enhanced clarity.

\section{References}

[1] R. Atkins, M. Barnsley, A. Vince, D. Wilson, A characterization of hyperbolic affine iterated function systems, Topology Proc., 36 (2010) 189211.

[2] T. Banakh, W. Kubiś, N. Novosad, M. Nowak, F. Strobin, Contractive function systems, their attractor and metrization, Topol. Methods Nonlinear Anal., 46 (2015), 1029-1066.

[3] M. Barnsley, Fractals Everywhere, Academic Press, Boston, MA, 1988.

[4] M. Barnsley, A. Vince, Real projective iterated function systems, J. Geom. Anal., 22 (2012), 1137-1172.

[5] D. Dumitru, Attractors of infinite iterated function systems containing contraction type functions, An. Ştiinţ. Univ. Al. I. Cuza Iaşi Mat. (N.S.), 59 (2013), 281-298.

[6] A. Edalat, Power domains and iterated function systems, Inf. Comput., 124 (1996), 182-197.

[7] R. Engelking, General Topology (Revised and completed edition), Heldermann Verlag, Berlin, 1989.

[8] A. Granas, J. Dugundji, Fixed Point Theory, Springer-Verlag, New York, 2003.

[9] J. Hutchinson, Fractals and self similarity, Indiana Univ. Math. J., 30 (1981), 713-747.

[10] J. Jachymski, I. Jóźwik, Nonlinear contractive conditions: a comparison and related problems, Banach Center Publ. , 77 (2007), 123-146. 
[11] A. Kameyama, Distances on topological self-similar sets and the kneading determinants, J. Math. Kyoto Univ., 40 (2000), 603-674.

[12] B. Kieninger, Iterated function systems on compact Hausdorff spaces, Ph.D. diss., University of Augsburg, Shaker-Verlag, Aachen, 2002.

[13] R. Miculescu, A. Mihail, Alternative characterization of hyperbolic infinite iterated function systems, J. Math. Anal. Appl., 407 (2013), 56-68.

[14] R. Miculescu, A. Mihail, On a question of A. Kameyama concerning self-similar metrics. J. Math. Anal. Appl., 422, 265-271 (2015).

[15] R. Miculescu, A. Mihail, A sufficient condition for a finite family of continuous functions to be transformed into $\psi$-contractions, Ann. Acad. Sci. Fenn., Math., 41 (2016), 51-65.

[16] R. Miculescu, A. Mihail, Remetrization results for possibly infinite self-similar systems, Topol. Methods Nonlinear Anal., 47 (2016), 333-345.

[17] R. Miculescu, A. Mihail, A generalization of Istrăţescu's fixed point theorem for convex contractions, Fixed Point Theory, 18 (2017), 689-702.

[18] R. Miculescu, A. Mihail, A generalization for a finite family of functions of the converse of Browder's fixed point theorem, Bull. Braz. Math. Soc. (N.S.), 49 (2018), 673-698.

[19] A. Mihail, A topological version od iterated function systems, An. Ştiinţ. Al. I. Cuza, Iaşi, (S.N.), Matematica, 58 (2012), 105-120.

[20] M. Samuel, A. Tetenov, On attractors of iterated function systems in uniform spaces.,Sib. Èlektron. Mat. Izv., 14 (2017), 151-155.

[21] A. Tetenov, Semigroups satisfying P-condition and topological selfsimilar sets, Sib. Ėlektron. Mat. Izv., 7 (2010), 461-464.

[22] S. Urziceanu, Alternative characterizations of AGIFSs having attractor, Fixed Point Theory, 20 (2019), 729-740.

[23] A. Vince, Mobius iterated function systems, Trans. Amer. Math. Soc., 365 (2013), 491-509.

Radu Miculescu

Faculty of Mathematics and Computer Science

Transilvania University of Braşov

Iuliu Maniu Street, nr. 50, 500091, Braşov, Romania

E-mail: radu.miculescu@unitbv.ro

Alexandru Mihail

Faculty of Mathematics and Computer Science

University of Bucharest 
Academiei Street 14, 010014, Bucharest, Romania E-mail: mihail_alex@yahoo.com 